Assiut Scientific Nursing Journal

http://asnj.journals.ekb.eg

http://www.arabimpactfactor.com

\title{
Effect of Cognitive Behavioral Therapy on Internet Gaming Disorder and Quality of Life Among Preparatory School Students in Alexandria
}

\author{
Hanan Hosni El Sherbini ${ }^{1}$ \& Rasha Abd El Hakim Abdou ${ }^{2}$. \\ 1. Assistant Professor Community Health Nursing, Faculty of Nursing, Alexandria University. \\ 2. Assistant Professor Community Health Nursing, Faculty of Nursing, Alexandria University.
}

\section{Abstract}

Background: Internet Gaming Disorder poses a serious threat to adolescent's physical and mental health. Cognitivebehavioral therapy (CBT) is a promising intervention for treatment and management of such problem. Objective: Assess the effect of cognitive behavioral therapy on the internet gaming disorder and quality of life among preparatory school students in Alexandria. Settings: The study was carried out at 4 randomly selected governmental preparatory schools in Alexandria. Subjects: All students in the first and second grades in the previously selected settings (2080 students) were screened using internet gaming disorder scale. 200 students with internet gaming disorder from the previously selected schools were randomly selected to assess the effect of CBT application. Tools: Three tools were used for data collection. The first tool was students' basic data structured interview schedule. The second tool was Internet Gaming Disorder Scale (IGD-20). The third tool was Ped Quality of Life Inventory Short Form (Peds QLTM - SF 15). Results: More than one tenth (12.7\%) of the studied students had internet gaming disorder. Immediately, after the program application around one third of them had no disorder and increased to $48.5 \%$ in the follow up phase. Before the program application, 37.0\% of them had good quality of life, raised to $60.5 \%$ and $51.5 \%$ in the immediate evaluation and in the follow up phase respectively. Conclusion and Recommendations: Internet gaming disorder is a prevalent problem among adolescents that affect their quality of life and CBT is an effective intervention 2 in managing such problem. The study recommended regular screening and early interventions and monitoring of internet use either by self or involving family members.

\section{Keywords: Internet Gaming Disorders, Adolescents \& Cognitive Behavioral Therapy.}

\section{Introduction}

Nowadays, internet becomes an integral and even inescapable part of many people's daily lives; they turn to it to send messages, read news, conduct business and much more activities. But recent scientific reports have begun to focus on the preoccupation some people develop with certain aspects of the Internet, particularly online games. The "gamers" play compulsively, to the exclusion of other interests, and their persistent and recurrent online activity results in clinically significant impairment or disorder (Gentile, et al., 2011).

Gaming disorders is defined as "a pattern of persistent or recurrent gaming behavior ("digital gaming" or "video-gaming"), which may be online (i.e., over the internet) or offline (Potenza, 2015).

According to the Diagnostic and Statistical Manual of Mental Disorders (DSM-5), Internet Gaming Disorder is indicated by the support of at least five core symptoms (from nine) in over one 12-month period. More specifically, the diagnostic criteria of Internet Gaming Disorder, including the following nine clinical symptoms: (1) preoccupation with videogames (i.e. "preoccupation"); (2) experiencing unpleasant symptoms when playing videogames (i.e. "withdrawal"); (3) the need to spend an increased amount of time involved in video games (i.e. "tolerance"); (4) failed attempts to control participation in videogames (i.e. "lose control"); (5) losing interest in past hobbies and entertainment as a result of, and with the exception of, videogames (i.e., "surrender from other activities"); (6) continue to use videogames despite having knowledge of psychosocial problems (i.e. "continuation"); (7) deceiving family members, therapists or others regarding the number of videogames (i.e. "fraud") (8) using videogames to escape or eliminate negative feelings (i.e., "escape") and 3 (9) harm or lose relationships, work, or education or significant career opportunities because of participation with videogames (i.e. "negative consequences") (Zajac, et al., 2017, \& Wichstrom, et al., 2018).

International estimates of internet gaming disorder was ranged from $0.6 \%$ to $15 \%$ (Lee, \& Morgan, 2018, \& Muller, et al., 2014).

The impact of internet gaming disorder can be very serious, often impacting individual, family and the whole community. It results in experiencing physical, social, and mental or psychological problems which in turn affect the quality of life. It has been linked to physical problems like sleep disturbance, eating problems, limited physical activity, back strain, eyestrain, and impaired vision. (Weinstein, et al., 2014, \& Kuss, \& Griffiths, 2015). 
The Internet games addicts transfer the social lives into the internet world. It leads to many social issues such as disturbing family, social, and workplace relations, where it isolates the persons from family and society and keeping them away from social interactions. It has a negative effect on psychological health such as depression, suicidal ideation, social phobia, schizophrenia, obsessive-compulsive disorder, antisocial/aggressive behaviors, and selfinjurious behavior (Reda, et al., 2012 \& Al Gammal, 2019).

A number of literatures have identified many risk factors for digital games addiction and several negative consequences resulting from this problem. Such risk factors include socio-demographic, social, psychological and mental factors, and internet use practices. It produces physical, social, and psychological problems, in addition to academic performance and career difficulties (Wolfling, et al., 2014).

Therefore, this problem needs immediate action and treatment. Thus, there are different ways to treat internet addiction and gaming disorder; however, cognitive behavioral therapy (CBT) has been proposed as an effective treatment. There is a specialized type or model to treat this disorder called cognitive behavioral therapy for internet addiction (CBT-IA) (Lee, \& Kang, 2015, \& Stevens, et al., 2018).

Cognitive behavioral therapy (CBT) is a short-term and problem-focused type of behavioral treatment. The CBT in general helps addicts to realize addictive feelings and actions, while learning new coping skills and methods to prevent a relapse. It focuses on helping addicts consider the relationship between beliefs, thoughts, and feelings and following behavior patterns and actions (Stevens $M$ et al 2018).

Internet Gaming Disorder is an increasingly prevalent disorder especially among adolescents, which can have severe consequences on them and on their families. There is an urgent need to improve existing treatment programs; these are currently hampered by the lack of research in this area. So, the aim of this study was to assess the prevalence of internet gaming disorder among adolescents and identify the effect of cognitive behavioral therapy on it.

\section{Significance of the study}

Data generated from this study can play an important role in identifying the magnitude and prevalence of internet gaming disorder among preparatory school students in Alexandria. Additionally, it helps to highlight factors behind such problem and can assist in planning and implementing comprehensive strategies for combating such phenomenon.

\section{Aims of the study \\ The aim of the study is to}

1. Assess the effect of applying Cognitive Behavioral Therapy on internet gaming disorder and quality of life among preparatory school students in Alexandria.

Research hypothesis

1. Preparatory schools' students with internet gaming disorder who received cognitive behavioral therapy will show lower level of disorder.

2. Preparatory schools' students with internet gaming disorder who received cognitive behavioral therapy will show higher level of quality of life.

\section{Materials \& Method \\ Materials \\ Research design}

The Quasi-Experimental design was adopted to carry out this study.

\section{Settings:}

This study was conducted in 4 governmental preparatory schools covering Alexandria Governorate as follow:

- Alexandria Governate is divided into eight educational zones affiliated to the Ministry of Education namely, El Montazah, East, West, Middle, El-Ajmi, El Amria, El Gomrok and Borg El Arab. By using the proportional allocation method, $25 \%$ of the total districts was selected randomly. It was two districts namely East and El Montaza.

- By using the equal allocation method, two governmental preparatory schools (one for boys and one for girls) affiliated to the selected two zones were selected randomly. The selected schools were El Ramel School for boys and El Dehreya School for Girls from East zone and from El Montazah zone; Tarek Abn Ziad School for Boys and El Sedek School for Girls.

\section{Subjects}

1. All students in the first and second grades in the previously selected schools were screened by using Internet Gaming Disorder Scale (IGD-20). They were 2080 students (According to Alexandria directorate of educational affairs data 2019). Third grade students were excluded from the study as the CBT were implemented then evaluated immediately and after 3 months, so third grade students will leave the school before being assessed for the intervention's effect.

2. By using the equal allocation method, 50 students were randomly selected from the 1 st and 2 nd grades from those having internet gaming disorder in each school (total number was 200 students).

\section{Inclusion criteria}

- Have internet gaming disorder. 
- Willing to participate in the study.

Tools: In order to collect the necessary data for the study, three tools were used:

Tool (I): Students' basic data structured interview schedule: It was developed by the researchers to collect data from students regarding their personal and socio-demographic characteristics, life style, health, social and educational data and Students' internet use

Tool (II): The Arabic Version of Internet Gaming Disorder test (IGD- 20 test):

It is a self-reporting instrument developed by Pontes $\mathrm{H}$ et al 2014 and translated into Arabic by Hawi N and Samaha, M 2017. It is a valid and reliable tool to assess both online and/or offline gaming activities occurring over a 12- month period based on the nine criteria set by the DSM-5. The (IGD-20) test comprises 20 items rated on a 5-point Likert scale; 1 (Strongly disagree), 2 (Disagree), 3 (Neither agree or disagree), 4 (Agree), and 5 (Strongly agree). The total score is determined by summing up the scores of the 20 items. Internet gaming disorder is diagnosed when the IGD-20 test total score is $\geq 71$ (Pontes $\mathrm{H}$ et al 2014). The internet gaming disorder level was classified as mild level is scored from 71 to 80 , moderate level is scored from 81 to 90 and severe level is ranged from 91 to 100 .

Tool (III): Pediatric Quality of Life InventoryShort Form (Peds QLTM - SF 15):

It is a 15 items self-reporting instrument developed by Chen, et al (2007) to measure the quality of life of the adolescent. The Peds QLTM- SF 15 items encompass the following subscales; physical functioning (5 items), emotional functioning (4 items), social functioning (3 items), and school functioning (3 items) and forming two domains; physical health (physical functioning subscale) and psychosocial health (sum of emotional functioning, social functioning, and school subscales). The items are rated on 5 points Likert scale, 7

ranging from 0 (never) to 4 (always). The total score ranges from 0 to 60 , with high scores meaning better quality of life.

\section{Methods}

Administrative process

- Before conduction of the study an official letter from the Faculty of Nursing, University of Alexandria was directed to the Central Agency for Public Mobilization and Statistics (CAPMAS), the Directorate of Education in Alexandria to obtain their approval to carry out the study at selected schools in Alexandria.

- Directors of the selected schools were met to explain the purpose of the study and the time for starting of the study in order to facilitate data collection and implementation of the nursing intervention.

I. Development of study tools

- Tool I was developed by the researchers after reviewing the current relevant literature to collect the necessary data.

- The content of the constructed tool was revised by a group of five experts in the field of the study to test its validity, completeness, and clarity of items, recommendations and suggestions of the jury were considered and the tool was modified accordingly.

- Using Cronbach Alpha Coefficient test. The reliability coefficient for tool II was $r=0.872$, and $r$ $=0.884$ for tool III.

\section{Pilot study}

A pilot study was carried out on a random sample of 20 students that were not included in the study sample in order to ascertain the relevance, clarity and applicability of the tools, test wording of the questions and estimate the time required for the interview. Based on the obtained results, the necessary modifications were done.

III. Nursing intervention (Cognitive Behavioral Therapy):

Preparation phase

- Initial assessment of all students in the first and second grades in the selected schools using the Arabic Version of the Internet Gaming Disorder Scale (IGD-20) was carried out before applying the cognitive behavioral therapy (CBT).

- Based on the results of the (IGD-20) analysis, from the students with internet gaming disorder, 50 students were selected randomly from the first and the second grade in each school, forming a total number of 200 students. They were further divided into 24 subgroups according to their educational grade and sex. Each group ranged from 8 to 9 students. (12 groups of boys and 12 groups of girls).

- The researcher selected well equipped, comfortable, safe, easily accessible and nonthreatening place that will provide privacy for all participants at the selected schools as library, lecturer room and sometimes computer room for conduction of the program sessions.

\section{Developmental phase}

The program objectives and methodology were prepared using CBT manual guide for children and adolescents. (Young, 2011) 


\section{Implementation phase}

The program was conducted on 14 consecutive sessions, 2 days per week, four sessions per day for four different groups. Each session duration ranged from 45-60 minutes.

Session 1 involved an introduction to the program, self-introduction, and an overview of treatment goals. Session 2, students described their own lives, including their psychological status (mood and anxiety), social environment, and family cohesion as well as their history of internet gaming. Sessions 3-5 dealt with students' "stress" in the following ways: (a) Defense and coping strategies were discussed; in response to stress, most students indulged in excessive internet gaming as an avoidance defense mechanism; (b) considering the pros and cons of internet gaming, students were asked to discuss the following: How can someone turn the cons into pros? How can internet gaming be turned into a healthy hobby? In responding to these questions, students were able to look at themselves objectively and reflect on their impulsive internet gaming; (c) students were educated on brain activity in response to stress associated with excessive and continuous internet use. Session 6, students were asked to introspect on their "self-identity," using to-do and not-to-do lists. During Sessions 7-9, the five steps of change; precontemplation, contemplation, preparation, action, and maintenance were discussed. In session 10, students' behavior patterns including impulsivity, emotions, and loneliness, as well as their thinking patterns, were discussed. During sessions 11 and 12, hurtful events provoking anxiety, depressed mood, and family conflicts were discussed. Session 13 covered the restoration of family cohesion and the social environment. In session 14, the students' changes were discussed and reinforced and termination of the program was done. The program was ended by simple party for the participants who shared in the program, giving them simple gifts and reward.

\section{Evaluation phase}

- The students were evaluated to determine the extent to which they have acquired the desired skills and practiced it.

- Evaluation of the students prior the program was done in the form of pretest administered to them using tool (II, and III). At the end of the program, a post test was carried out using the same tools as in pretest. Post tests were conducted twice, immediately after the end of the program and 3 months later to evaluate the immediate and retained changes in students' behaviors.

\section{Ethical considerations}

- Informed oral consents were obtained from the students after brief explanation of the purpose and nature of the research.

- The anonymity and confidentiality of responses, voluntary participation and right to refuse to participate in the study were emphasized. The researcher explained the objectives of the study to the participants. Privacy was ensured.

- Data was collected by the researchers during the period from September 2018 to May 2019 (9 months) during the academic year 2018-2019.

\section{Statistical analysis}

After data were collected, they were coded and transferred into specially designed formats so as to be suitable for computer feeding. Following data entry, checking and verification processes were carried out to avoid any errors during data entry, frequency analysis, cross tabulation and manual revision were all used to detect any errors. The statistical package for social sciences (SPSS version 20) was utilized for both data presentation and statistical analysis of the results. The level of significance selected for this study was $\mathrm{P}$ equal to or less than 0.05 . 
Results

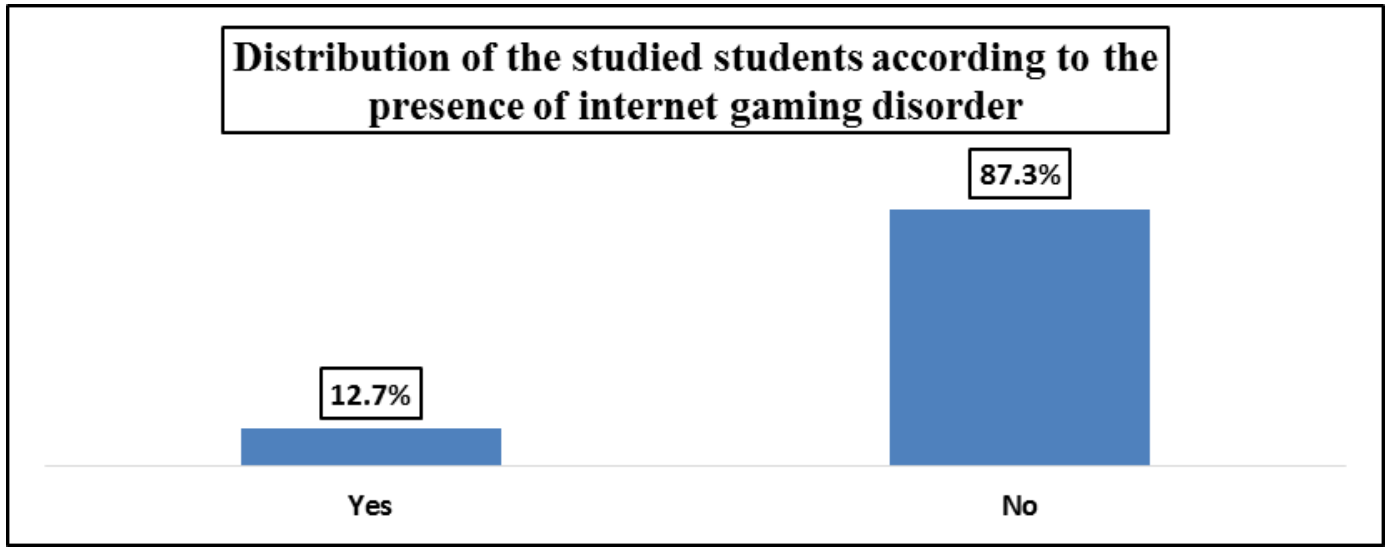

Figure (1): Distribution of the studied students according to the presence of internet gaming disorder.

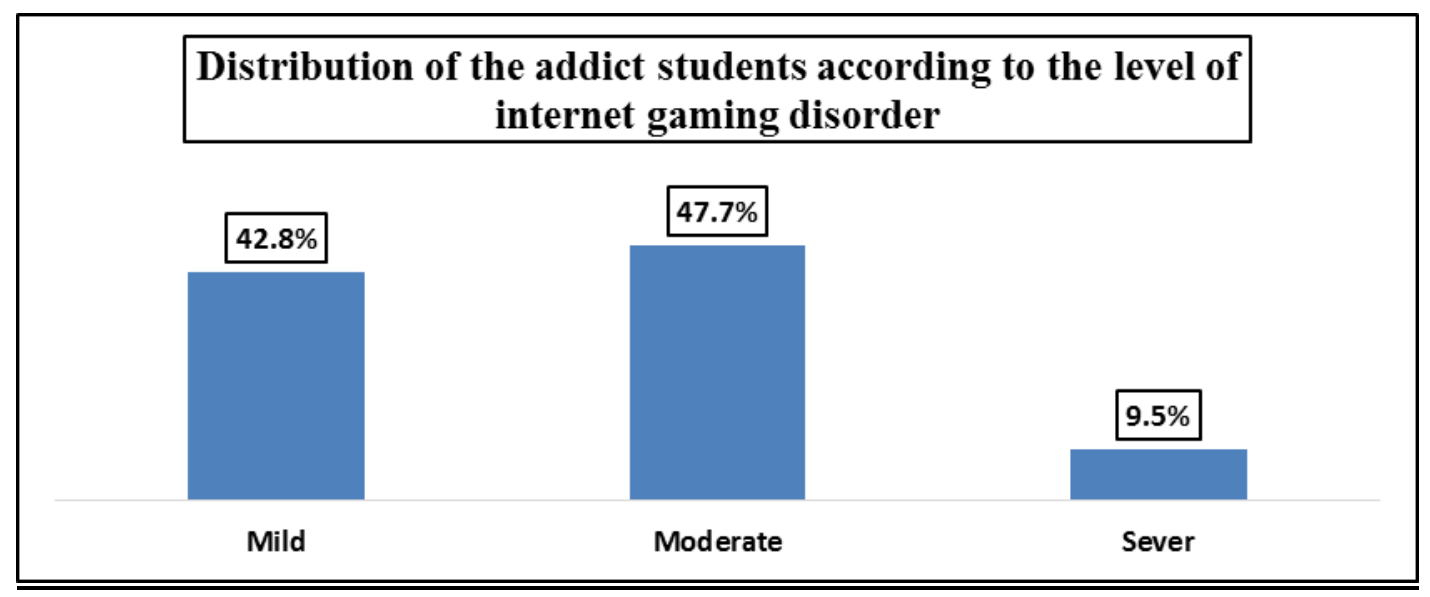

Figure (2): Distribution of the internet gaming disorder students according to the level of internet gaming disorder.

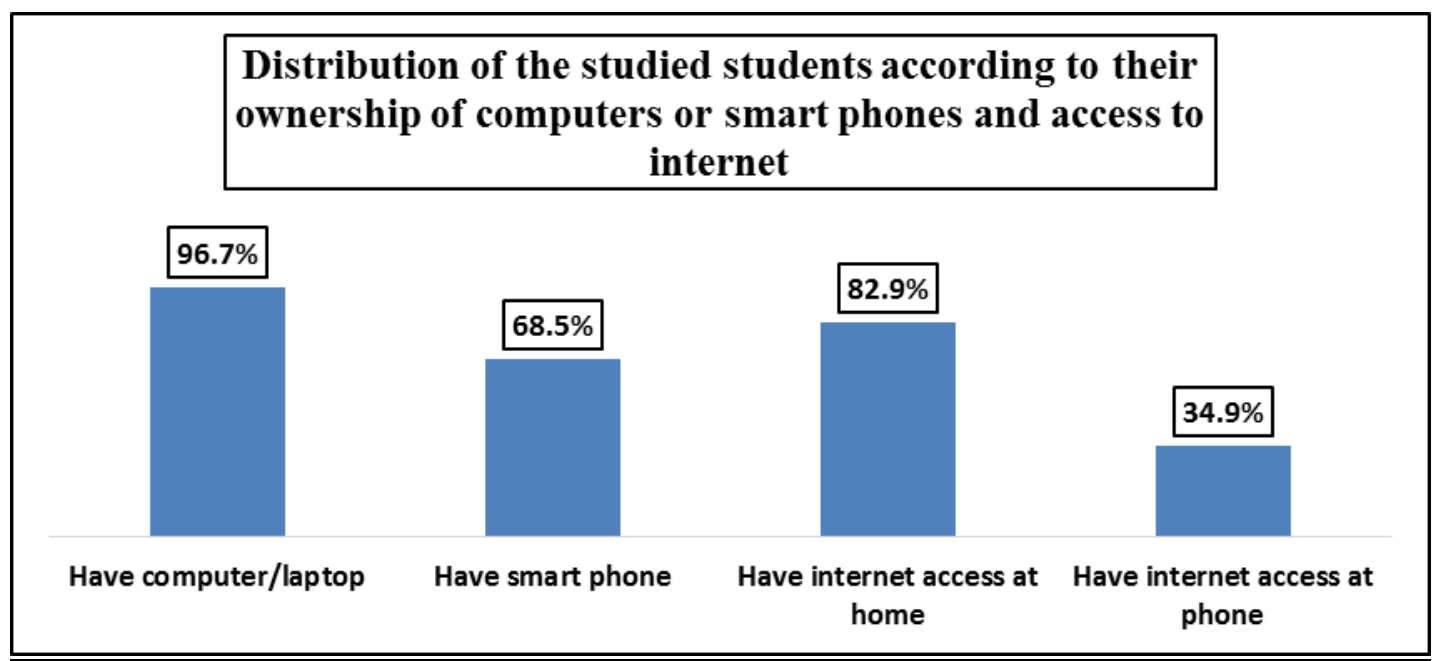

Figure (3): Distribution of the students according to the ownership of computer or smart phone and access to internet 


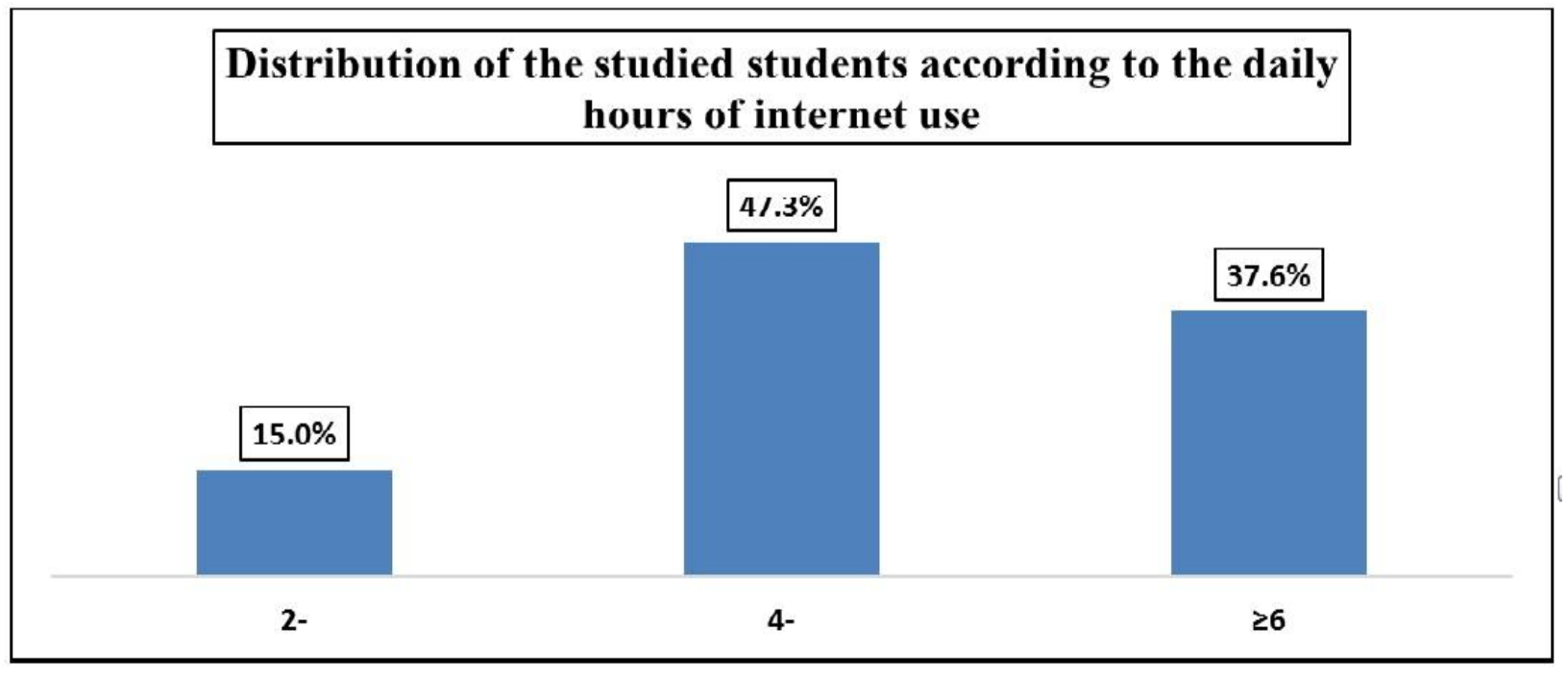

Figure (4): Distribution of the students according to the daily hours of internet use

Table (I): Distribution of the students according to the presence of internet gaming disorder and their basic characteristics.

\begin{tabular}{|c|c|c|c|c|c|c|c|}
\hline \multirow{3}{*}{ Students' characteristics } & \multicolumn{4}{|c|}{ Internet gaming disorder } & \multirow{2}{*}{\multicolumn{2}{|c|}{$\begin{array}{c}\text { Total } \\
\mathbf{N}=\mathbf{2 0 8 0}\end{array}$}} & \multirow{3}{*}{ Test of Significance } \\
\hline & \multicolumn{2}{|c|}{$\begin{array}{c}\text { No } \\
N=1816\end{array}$} & \multicolumn{2}{|c|}{$\begin{array}{c}\text { Yes } \\
\mathrm{N}=264\end{array}$} & & & \\
\hline & No. & $\%$ & No. & $\%$ & No. & $\%$ & \\
\hline \multicolumn{8}{|l|}{ Age (years) } \\
\hline $\begin{array}{l}-12- \\
-14- \\
-\geq 16\end{array}$ & $\begin{array}{c}916 \\
869 \\
31\end{array}$ & $\begin{array}{l}85.0 \\
89.8 \\
91.2\end{array}$ & $\begin{array}{c}162 \\
99 \\
3\end{array}$ & $\begin{array}{c}15.0 \\
10.2 \\
8.8\end{array}$ & $\begin{array}{c}1078 \\
968 \\
34\end{array}$ & $\begin{array}{c}51.8 \\
46.5 \\
1.6\end{array}$ & $\begin{array}{c}X^{2}=11.074 \\
P=0.004^{*}\end{array}$ \\
\hline \multicolumn{8}{|l|}{ Sex } \\
\hline $\begin{array}{l}\text { - Male } \\
\text { - Female }\end{array}$ & $\begin{array}{l}891 \\
925\end{array}$ & $\begin{array}{l}86.5 \\
88.1\end{array}$ & $\begin{array}{l}139 \\
125\end{array}$ & $\begin{array}{l}13.5 \\
11.9\end{array}$ & $\begin{array}{l}1030 \\
1050\end{array}$ & $\begin{array}{l}49.5 \\
50.5\end{array}$ & $\begin{array}{l}X^{2}=1.187 \\
P=0.276\end{array}$ \\
\hline \multicolumn{8}{|l|}{ Educational years } \\
\hline $\begin{array}{l}\text { - First } \\
\text { - Second } \\
\end{array}$ & $\begin{array}{l}991 \\
824 \\
\end{array}$ & $\begin{array}{l}80.0 \\
97.9 \\
\end{array}$ & $\begin{array}{c}247 \\
18 \\
\end{array}$ & $\begin{array}{c}20.0 \\
2.1 \\
\end{array}$ & $\begin{array}{c}1238 \\
842 \\
\end{array}$ & $\begin{array}{l}59.5 \\
40.5 \\
\end{array}$ & $\begin{aligned} \mathrm{X}^{2} & =143.049 \\
\mathrm{P} & =0.000^{*}\end{aligned}$ \\
\hline \multicolumn{8}{|l|}{ Birth order } \\
\hline $\begin{array}{l}\text { - First } \\
\text { - Second } \\
\text { - Third } \\
\text { - Forth and more }\end{array}$ & $\begin{array}{l}642 \\
573 \\
360 \\
241\end{array}$ & $\begin{array}{l}89.5 \\
87.7 \\
86.1 \\
82.5\end{array}$ & $\begin{array}{l}75 \\
80 \\
58 \\
51\end{array}$ & $\begin{array}{l}10.5 \\
12.3 \\
13.9 \\
17.5\end{array}$ & $\begin{array}{l}717 \\
653 \\
418 \\
292\end{array}$ & $\begin{array}{l}34.5 \\
31.4 \\
20.1 \\
14.0\end{array}$ & $\begin{array}{l}X^{2}=9.871 \\
P=0.019^{*}\end{array}$ \\
\hline \multicolumn{8}{|l|}{ Place of residence } \\
\hline $\begin{array}{l}\text { - Urban } \\
\text { - Sub-urban } \\
\text { - Rural }\end{array}$ & $\begin{array}{l}527 \\
574 \\
715\end{array}$ & $\begin{array}{l}79.1 \\
93.3 \\
89.5\end{array}$ & $\begin{array}{c}139 \\
41 \\
84\end{array}$ & $\begin{array}{c}20.9 \\
6.7 \\
10.5\end{array}$ & $\begin{array}{l}666 \\
615 \\
799\end{array}$ & $\begin{array}{l}32.0 \\
29.6 \\
38.4\end{array}$ & $\begin{array}{c}X^{2}=63.776 \\
P=0.000^{*}\end{array}$ \\
\hline
\end{tabular}

$X^{2}$ Chi Square test $\quad *$ Statistically significant at $p \geq 0.05$ 
Table (2): Distribution of the students according to the presence of internet gaming disorder and their families' characteristics: $(\mathrm{N}=\mathbf{2 0 8 0})$

\begin{tabular}{|c|c|c|c|c|c|c|c|}
\hline \multirow{3}{*}{ Items } & \multicolumn{4}{|c|}{ Internet gaming disorder } & \multirow{2}{*}{\multicolumn{2}{|c|}{$\begin{array}{c}\text { Total } \\
\mathbf{N}=\mathbf{2 0 8 0}\end{array}$}} & \multirow{3}{*}{$\begin{array}{c}\text { Test of } \\
\text { Significance }\end{array}$} \\
\hline & \multicolumn{2}{|c|}{$\begin{array}{c}\text { No } \\
\mathrm{N}=1816\end{array}$} & \multicolumn{2}{|c|}{$\begin{array}{c}\text { Yes } \\
\mathrm{N}=264\end{array}$} & & & \\
\hline & No. & $\%$ & No. & $\%$ & No. & $\%$ & \\
\hline \multicolumn{8}{|l|}{ Parents' marital status } \\
\hline - Married (living together) & 1648 & 90.2 & 179 & 9.8 & 1827 & 87.8 & \\
\hline - Divorced / separated & 86 & 72.3 & 33 & 27.7 & 119 & 5.7 & $X^{2}=126.128$ \\
\hline - Father died & 73 & 64.0 & 41 & 36.0 & 114 & 5.5 & $\mathrm{P}=0.000 *$ \\
\hline - Mother died & 9 & 45.0 & 11 & 55.0 & 20 & 1.0 & \\
\hline Fathers' age (years) & \multicolumn{2}{|c|}{$\mathrm{N}=1743$} & \multicolumn{2}{|c|}{$\mathrm{N}=223$} & \multicolumn{2}{|c|}{$\mathrm{N}=1966$} & \\
\hline$-30-$ & 166 & 91.7 & 15 & 8.3 & 181 & 9.2 & \\
\hline$-40-$ & 1178 & 91.5 & 109 & 8.5 & 1287 & 65.5 & $X^{2}=51.853$ \\
\hline$-50-$ & 339 & 81.3 & 78 & 18.7 & 417 & 21.2 & $\mathrm{P}=0.000^{*}$ \\
\hline$-\geq 60$ & 60 & 74.1 & 21 & 25.9 & 81 & 4.1 & \\
\hline Fathers' education & & & & & \multicolumn{2}{|c|}{$\mathrm{N}=1966$} & \\
\hline - Illiterate / Read and write & 725 & 98.1 & 14 & 1.9 & 739 & 37.6 & \\
\hline - Basic education & 395 & 88.8 & 50 & 11.2 & 445 & 22.6 & $X^{2}=159.118$ \\
\hline - Secondary / technical education & 498 & 83.1 & 101 & 16.9 & 599 & 30.5 & $\mathrm{P}=0.000^{*}$ \\
\hline - University education & 125 & 68.3 & 58 & 31.7 & 183 & 9.3 & \\
\hline Fathers' occupation & & & & & \multicolumn{2}{|c|}{$\mathrm{N}=1966$} & \\
\hline - Working & 1662 & 88.6 & 213 & 11.4 & 1875 & 96.9 & $X^{2}=0.012$ \\
\hline - Not work / on retirement & 81 & 89.0 & 10 & 11.0 & 91 & 3.1 & $P=0.913$ \\
\hline Mothers' age (years) & \multicolumn{2}{|c|}{$\mathrm{N}=1807$} & \multicolumn{2}{|c|}{$\mathrm{N}=\mathbf{2 5 3}$} & \multicolumn{2}{|c|}{$\mathrm{N}=\mathbf{2 0 6 0}$} & \\
\hline$-25-$ & 377 & 88.1 & 51 & 11.9 & 428 & 20.8 & \\
\hline$-35-$ & 1172 & 87.9 & 162 & 12.1 & 1334 & 64.8 & 04 \\
\hline$-\geq 45$ & 258 & 86.6 & 40 & 13.4 & 298 & 14.5 & \\
\hline Mothers' education & & & & & \multicolumn{2}{|c|}{$\mathrm{N}=2060$} & \\
\hline - Illiterate/ Read and write & 753 & 92.4 & 62 & 7.6 & 815 & 39.6 & \\
\hline - Basic education & 359 & 87.1 & 53 & 12.9 & 412 & 20.0 & $X^{2}=35.713$ \\
\hline - Secondary / technical education & 586 & 84.6 & 107 & 15.4 & 693 & 33.6 & $\mathrm{P}=0.000^{*}$ \\
\hline - University education & 109 & 77.9 & 31 & 22.1 & 140 & 6.8 & \\
\hline Mothers' occupation & & & & & \multicolumn{2}{|c|}{$\mathrm{N}=2060$} & \\
\hline - Working & 226 & 71.7 & 89 & 28.3 & 315 & 15.3 & $\mathrm{X}^{2}=88.060$ \\
\hline - Not work / on retirement & 1581 & 90.6 & 164 & 9.4 & 1745 & 84.7 & $\mathrm{P}=0.000 *$ \\
\hline Sufficiency of income & \multicolumn{2}{|c|}{$\mathrm{N}=1816$} & \multicolumn{2}{|c|}{$N=264$} & \multicolumn{2}{|c|}{$\mathrm{N}=\mathbf{2 0 8 0}$} & \\
\hline - Not enough & 676 & 91.1 & 66 & 8.9 & 742 & 35.7 & $\mathrm{X}^{2}=15.011$ \\
\hline - Enough & 1140 & 85.2 & 198 & 14.8 & 1338 & 64.3 & $\mathrm{P}=0.000^{*}$ \\
\hline \multicolumn{8}{|l|}{ Socio-economic level } \\
\hline - Low & 962 & 93.1 & 71 & 6.9 & 1033 & 49.7 & \\
\hline - Middle & 787 & 82.0 & 173 & 18.0 & 960 & 46.2 & $\begin{array}{l}\lambda=04.48 l \\
P=0.000 *\end{array}$ \\
\hline - High & 67 & 77.0 & 20 & 23.0 & 87 & 4.2 & \\
\hline \multicolumn{8}{|c|}{ Parents' supervision on the students' activities } \\
\hline - Yes & 1444 & 97.1 & 43 & 2.9 & 1487 & 71.5 & $\mathrm{X}^{2}=452.095$ \\
\hline - No & 372 & 62.7 & 221 & 37.3 & 593 & 28.5 & $\mathrm{P}=0.000^{*}$ \\
\hline
\end{tabular}

$X^{2}$ Chi Square test $\quad *$ Statistically significant at $p \geq 0.05$ 
Table (3): Distribution of the students according to the presence of internet gaming disorder and their life style habits: $(\mathrm{N}=\mathbf{2 0 8 0})$.

\begin{tabular}{|c|c|c|c|c|c|c|c|}
\hline \multirow{3}{*}{ Items } & \multicolumn{4}{|c|}{ Internet gaming disorder } & \multirow{2}{*}{\multicolumn{2}{|c|}{$\begin{array}{c}\text { Total } \\
\mathbf{N}=\mathbf{2 0 8 0}\end{array}$}} & \multirow{3}{*}{$\begin{array}{c}\text { Test of } \\
\text { Significance }\end{array}$} \\
\hline & \multicolumn{2}{|c|}{$\begin{array}{c}\text { No } \\
\mathrm{N}=1816\end{array}$} & \multicolumn{2}{|c|}{$\begin{array}{c}\text { Yes } \\
N=264\end{array}$} & & & \\
\hline & No. & $\%$ & No. & $\%$ & No. & $\%$ & \\
\hline \multicolumn{8}{|c|}{ Regular meals per day } \\
\hline - Yes & 510 & 93.9 & 33 & 6.1 & 543 & 26.1 & $X^{2}=29.017$ \\
\hline - No & 1306 & 85.0 & 231 & 15.0 & 1537 & 73.9 & $\mathrm{P}=0.000 *$ \\
\hline \multicolumn{8}{|c|}{ Skipped /Missed meals } \\
\hline- Yes & 590 & 79.8 & 149 & 20.2 & 739 & 35.5 & $X^{2}=57.721$ \\
\hline - No & 1226 & 91.4 & 115 & 8.6 & 1341 & 64.5 & $\mathrm{P}=0.000^{*}$ \\
\hline \multicolumn{8}{|c|}{ Practicing of exercises } \\
\hline- Yes & 866 & 90.3 & 93 & 9.7 & 959 & 46.1 & $X^{2}=14.401$ \\
\hline - No & 950 & 84.7 & 171 & 15.3 & 1121 & 53.9 & $\mathrm{P}=0.000^{*}$ \\
\hline \multicolumn{8}{|c|}{ Sleeping hours per day } \\
\hline$-5-$ & 942 & 86.3 & 149 & 13.7 & 1091 & 52.5 & \\
\hline$-7-$ & 521 & 87.4 & 75 & 12.6 & 596 & 28.7 & $X^{2}=3.326$ \\
\hline$-9-$ & 190 & 89.2 & 23 & 10.8 & 213 & 10.2 & $P=0.344$ \\
\hline$-\geq 11$ & 163 & 90.6 & 17 & 9.4 & 180 & 8.7 & \\
\hline \multicolumn{8}{|c|}{ Having sleeping problems } \\
\hline - Yes & 664 & 77.7 & 191 & 22.3 & 855 & 41.1 & $\mathrm{X}^{2}=121.919$ \\
\hline - No & 1152 & 94.0 & 73 & 6.0 & 1225 & 58.9 & $\mathrm{P}=0.000^{*}$ \\
\hline
\end{tabular}

$X^{2}$ Chi Square test * Statistically significant at $p \geq 0.05$

Table (4): Distribution of the students according to the presence of internet gaming disorder and their health status related data: $(\mathrm{N}=\mathbf{2 0 8 0})$.

\begin{tabular}{|c|c|c|c|c|c|c|c|}
\hline \multirow{3}{*}{ Items } & \multicolumn{4}{|c|}{ Internet gaming disorder } & \multirow{2}{*}{\multicolumn{2}{|c|}{$\begin{array}{c}\text { Total } \\
\mathrm{N}=2080\end{array}$}} & \multirow{3}{*}{$\begin{array}{c}\text { Test of } \\
\text { Significance }\end{array}$} \\
\hline & \multicolumn{2}{|c|}{$\begin{array}{c}\text { No } \\
\mathrm{N}=\mathbf{1 8 1 6}\end{array}$} & \multicolumn{2}{|c|}{$\begin{array}{c}\text { Yes } \\
N=264\end{array}$} & & & \\
\hline & No. & $\%$ & No. & $\%$ & No. & $\%$ & \\
\hline \multicolumn{8}{|l|}{ Having chronic diseases } \\
\hline - Yes & 616 & 89.1 & 75 & 10.9 & 691 & 33.2 & $X^{2}=3.156$ \\
\hline - No & 1200 & 86.4 & 189 & 13.6 & 1389 & 66.8 & $\mathrm{P}=0.076$ \\
\hline \multicolumn{8}{|l|}{ Have current health complaints } \\
\hline - Yes & 189 & 44.0 & 241 & 56.0 & 430 & 20.77 & $\mathrm{X}^{2}=919.426$ \\
\hline$-\mathrm{No}$ & 1627 & 98.6 & 23 & 1.4 & 1650 & 9.3 & $\mathrm{P}=0.000^{*}$ \\
\hline Current health complaints \# & \multicolumn{2}{|c|}{$\mathrm{N}=189$} & \multicolumn{2}{|c|}{$\mathrm{N}=241$} & \multicolumn{2}{|c|}{$\mathrm{N}=430$} & \\
\hline - Vision Problems & 72 & 41.6 & 101 & 58.4 & 173 & 40.2 & \\
\hline - Neck \& shoulder pain & 136 & 47.4 & 153 & 52.9 & 289 & 67.2 & $X=14.983$ \\
\hline - Headache & 32 & 26.4 & 89 & 73.6 & 121 & 28.1 & $P=0.001^{*}$ \\
\hline
\end{tabular}

\# Multiple answers are allowed $\quad X^{2}$ Chi Square test $\quad *$ Statistically significant at $p \geq 0.05$ 
Table (5): Distribution of the students according to the presence of internet gaming disorder and their perspective about their social relations: $(N=2080)$

\begin{tabular}{|c|c|c|c|c|c|c|c|}
\hline \multirow{3}{*}{ Items } & \multicolumn{4}{|c|}{ internet gaming disorder } & \multirow{2}{*}{\multicolumn{2}{|c|}{$\begin{array}{c}\text { Total } \\
\mathbf{N}=\mathbf{2 0 8 0}\end{array}$}} & \multirow{3}{*}{ Test of Significance } \\
\hline & \multicolumn{2}{|c|}{$\begin{array}{c}\text { No } \\
\mathrm{N}=\mathbf{1 8 1 6}\end{array}$} & \multicolumn{2}{|c|}{$\begin{array}{c}\text { Yes } \\
N=264\end{array}$} & & & \\
\hline & No. & $\%$ & No. & $\%$ & No. & $\%$ & \\
\hline \multicolumn{8}{|c|}{ Have problems in relations with family members } \\
\hline $\begin{array}{l}\text { - Yes } \\
- \text { No }\end{array}$ & $\begin{array}{c}16 \\
1800\end{array}$ & $\begin{array}{l}45.7 \\
88.0\end{array}$ & $\begin{array}{c}19 \\
245\end{array}$ & $\begin{array}{l}54.3 \\
12.0\end{array}$ & $\begin{array}{c}35 \\
2045\end{array}$ & $\begin{array}{c}1.7 \\
98.3\end{array}$ & $\begin{aligned} X^{2} & =55.5773 \\
P & =0.000^{*}\end{aligned}$ \\
\hline \multicolumn{8}{|c|}{ Have problems in relations with friends } \\
\hline $\begin{array}{l}\text { - Yes } \\
\text { - No }\end{array}$ & $\begin{array}{c}49 \\
1767\end{array}$ & $\begin{array}{l}25.0 \\
93.8\end{array}$ & $\begin{array}{l}147 \\
117\end{array}$ & $\begin{array}{c}75.0 \\
6.2\end{array}$ & $\begin{array}{c}196 \\
1884\end{array}$ & $\begin{array}{c}9.4 \\
90.6\end{array}$ & $\begin{aligned} \mathrm{X}^{2} & =758.103 \\
\mathrm{P} & =0.000^{*}\end{aligned}$ \\
\hline \multicolumn{8}{|c|}{ Have problems in relations with school personnel } \\
\hline $\begin{array}{l}\text { - Yes } \\
\text { - No } \\
\end{array}$ & $\begin{array}{c}28 \\
1788\end{array}$ & $\begin{array}{l}39.4 \\
89.0\end{array}$ & $\begin{array}{c}43 \\
221\end{array}$ & $\begin{array}{l}60.6 \\
11.0\end{array}$ & $\begin{array}{c}71 \\
2009\end{array}$ & $\begin{array}{c}3.4 \\
96.6\end{array}$ & $\begin{aligned} \mathrm{X}^{2} & =152.018 \\
\mathrm{P} & =0.000^{*}\end{aligned}$ \\
\hline
\end{tabular}

$X^{2}$ Chi Square test $\quad *$ Statistically significant at $p \geq 0.05$

Table (6): Distribution of the students according to the presence of internet gaming disorder and their scholastic achievement and leisure time activities: $(N=2080)$

\begin{tabular}{|c|c|c|c|c|c|c|c|}
\hline \multirow{3}{*}{ Items } & \multicolumn{4}{|c|}{$\begin{array}{c}\text { Internet gaming } \\
\text { disorder }\end{array}$} & \multirow{2}{*}{\multicolumn{2}{|c|}{$\begin{array}{c}\text { Total } \\
\mathbf{N}=\mathbf{2 0 8 0}\end{array}$}} & \multirow{3}{*}{$\begin{array}{c}\text { Test of } \\
\text { Significance }\end{array}$} \\
\hline & \multicolumn{2}{|c|}{$\begin{array}{c}\text { No } \\
\mathrm{N}=1816\end{array}$} & \multicolumn{2}{|c|}{$\begin{array}{c}\text { Yes } \\
\mathrm{N}=264\end{array}$} & & & \\
\hline & No. & $\%$ & No. & $\%$ & No. & $\%$ & \\
\hline \multicolumn{8}{|c|}{ Previous school failure } \\
\hline - Yes & 166 & 49.0 & 173 & 51.0 & 339 & 16.3 & $X^{2}=537.253$ \\
\hline - No & 1650 & 94.8 & 91 & 5.2 & 1741 & 83.7 & $\mathrm{P}=0.000 *$ \\
\hline \multicolumn{8}{|c|}{ Academic performance in the previous year } \\
\hline - Excellent & 340 & 96.3 & 13 & 3.7 & 353 & 17.0 & \\
\hline - Very good & 545 & 95.1 & 28 & 4.9 & 573 & 27.5 & $X^{2}=244.514$ \\
\hline - Good & 656 & 88.8 & 83 & 11.2 & 739 & 35.5 & $\mathrm{P}=0.000^{*}$ \\
\hline - Passable & 275 & 66.3 & 140 & 33.7 & 415 & 20.0 & \\
\hline \multicolumn{8}{|c|}{ Regular attendance to school } \\
\hline - Yes & 1607 & 98.2 & 30 & 1.8 & 1637 & 78.7 & $X^{2}=817.993$ \\
\hline - No & 209 & 47.2 & 234 & 52.8 & 443 & 21.3 & $\mathrm{P}=0.000 *$ \\
\hline \multicolumn{8}{|c|}{ Share in school activities } \\
\hline - Yes & 523 & 88.5 & 68 & 11.5 & 591 & 28.4 & $X^{2}=1.049$ \\
\hline - No & 1293 & 86.8 & 196 & 13.2 & 1489 & 71.6 & $\mathrm{P}=0.306$ \\
\hline \multicolumn{8}{|c|}{ Share in recreational activities } \\
\hline - Yes & 523 & 90.6 & 54 & 9.4 & 577 & 27.7 & $X^{2}=8.008$ \\
\hline - No & 1293 & 86.0 & 210 & 14.0 & 1503 & 72.3 & $P=0.005^{*}$ \\
\hline
\end{tabular}

$X^{2}$ Chi Square test $\quad *$ Statistically significant at $p \geq 0.05$ 
Table (7): The difference between internet gaming disorder and the quality of life among the students: $(\mathrm{N}=\mathbf{2 0 8 0})$.

\begin{tabular}{|c|c|c|c|c|c|c|c|}
\hline \multirow{3}{*}{ Items } & \multicolumn{4}{|c|}{ Internet gaming disorder } & \multirow{2}{*}{\multicolumn{2}{|c|}{$\begin{array}{c}\text { Total } \\
\mathbf{N}=\mathbf{2 0 8 0}\end{array}$}} & \multirow{3}{*}{ Test of Significanc } \\
\hline & \multicolumn{2}{|c|}{$\begin{array}{c}\text { No } \\
\mathrm{N}=1816\end{array}$} & \multicolumn{2}{|c|}{$\begin{array}{c}\text { Yes } \\
N=264\end{array}$} & & & \\
\hline & No. & $\%$ & No. & $\%$ & No. & $\%$ & \\
\hline \multicolumn{8}{|c|}{ Physical functioning } \\
\hline - Good & 863 & 93.1 & 64 & 6.9 & 927 & 44.6 & \multirow{3}{*}{$\begin{aligned} \mathrm{X}^{2} & =132.269 \\
\mathrm{P} & =0.000^{*}\end{aligned}$} \\
\hline - Fair & 790 & 87.3 & 115 & 12.7 & 905 & 43.5 & \\
\hline - Poor & 163 & 65.7 & 85 & 34.3 & 248 & 11.9 & \\
\hline \multicolumn{8}{|c|}{ Emotional functioning } \\
\hline - Good & 978 & 90.5 & 103 & 9.5 & 1081 & 52.0 & \multirow{3}{*}{$\begin{array}{c}X^{2}=87.411 \\
P=0.000^{*}\end{array}$} \\
\hline - Fair & 613 & 89.8 & 70 & 10.2 & 683 & 32.8 & \\
\hline - Poor & 225 & 71.2 & 91 & 28.8 & 316 & 15.2 & \\
\hline \multicolumn{8}{|l|}{ Social functioning } \\
\hline - Good & 868 & 90.9 & 87 & 9.1 & 955 & 45.9 & \multirow{3}{*}{$\begin{array}{l}X^{2}=51.686 \\
P=0.000^{*}\end{array}$} \\
\hline - Fair & 611 & 88.7 & 78 & 11.3 & 689 & 33.1 & \\
\hline - Poor & 337 & 77.3 & 99 & 22.7 & 436 & 21.0 & \\
\hline \multicolumn{8}{|l|}{ School functioning } \\
\hline - Good & 571 & 87.7 & 80 & 12.3 & 651 & 31.3 & \multirow{3}{*}{$\begin{array}{c}X^{2}=23.996 \\
P=0.000^{*}\end{array}$} \\
\hline - Fair & 896 & 90.0 & 100 & 10.0 & 996 & 47.9 & \\
\hline - Poor & 349 & 80.6 & 84 & 19.4 & 433 & 20.8 & \\
\hline \multicolumn{8}{|c|}{ Total Psychosocial functioning } \\
\hline - Good & 802 & 89.7 & 92 & 10.3 & 894 & 43.0 & \multirow{3}{*}{$\begin{array}{c}X^{2}=41.582 \\
P=0.000^{*}\end{array}$} \\
\hline - Fair & 700 & 89.5 & 82 & 10.5 & 782 & 37.6 & \\
\hline - Poor & 314 & 77.7 & 90 & 22.3 & 404 & 19.4 & \\
\hline \multicolumn{8}{|c|}{ Total student's quality of life } \\
\hline - Good & 817 & 90.9 & 82 & 9.1 & 899 & 43.2 & \multirow{3}{*}{$\begin{array}{c}X^{2}=53.988 \\
P=0.000^{*}\end{array}$} \\
\hline - Fair & 735 & 88.3 & 97 & 11.7 & 832 & 40.0 & \\
\hline - Poor & 264 & 75.6 & 85 & 24.4 & 349 & 16.8 & \\
\hline
\end{tabular}

$X^{2}$ Chi Square test $\quad *$ Statistically significant at $p \geq 0.05$

Table (8): Difference between levels of internet gaming disorder among the students across the study phases: $(\mathrm{N}=\mathbf{2 0 0})$.

\begin{tabular}{|c|c|c|c|c|c|c|c|}
\hline \multirow{3}{*}{ Items } & \multicolumn{6}{|c|}{ Total $(n=200)$} & \multirow{3}{*}{ Test of Significance } \\
\hline & \multicolumn{2}{|c|}{ Before } & \multicolumn{2}{|c|}{ Post 1} & \multicolumn{2}{|c|}{ Post 2} & \\
\hline & No. & $\%$ & No. & $\%$ & No. & $\%$ & \\
\hline \multicolumn{8}{|c|}{ Internet gaming disorder } \\
\hline- No & 0 & 0.0 & 65 & 32.5 & 97 & 48.5 & \multirow{5}{*}{$\begin{array}{ll}\mathrm{X}^{2 \mathrm{~b}}=137.61 & \mathrm{P}=0.000^{*} \\
\mathrm{X}^{2 \mathrm{c}}=197.76 & \mathrm{P}=0.000^{*} \\
\mathrm{X}^{2 \mathrm{~d}}=10.885 & \mathrm{P}=0.004^{*}\end{array}$} \\
\hline - Mild & 80 & 40.0 & 112 & 56.0 & 88 & 44.0 & \\
\hline - Moderate & 107 & 53.5 & 23 & 11.5 & 15 & 7.5 & \\
\hline \multirow[t]{2}{*}{ - Sever } & 13 & 6.5 & 0 & 0.0 & 0 & 0.0 & \\
\hline & \multicolumn{6}{|c|}{$\mathrm{X}^{2 \mathrm{a}}=229.90 \quad \mathrm{P}=0.000^{*}$} & \\
\hline
\end{tabular}

$X^{2}=$ Chi Square test $\quad X^{2 a}$ comparison of the group across the study phases

$X^{2 b}$ comparison between pre and post $1 \quad X^{2 c}$ comparison between pre and post 2

$X^{2 d}$ comparison between post 1 and post $2 *$ Significant $p$ at $\leq 0.05$ 
Table (9): Difference between levels of quality of life among the students across the study phases: $(N=$ 200).

\begin{tabular}{|c|c|c|c|c|c|c|c|}
\hline \multirow{3}{*}{ Items } & \multicolumn{6}{|c|}{ Total $(n=200)$} & \multirow{3}{*}{ Test of Significance } \\
\hline & \multicolumn{2}{|c|}{ Before } & \multicolumn{2}{|c|}{ Post 1} & \multicolumn{2}{|c|}{ Post 2} & \\
\hline & No. & $\%$ & No. & $\%$ & No. & $\%$ & \\
\hline \multicolumn{8}{|c|}{ Physical functioning } \\
\hline - Good & 54 & 27.0 & 118 & 59.0 & 111 & 55.5 & $\mathrm{X}^{2 \mathrm{~b}}=57.46 \quad \mathrm{P}=0.000^{*}$ \\
\hline - Fair & 86 & 43.0 & 70 & 35.0 & 80 & 40.0 & $\mathrm{X}^{2 \mathrm{c}}=57.60 \quad \mathrm{P}=0.000^{*}$ \\
\hline \multirow[t]{2}{*}{ - Poor } & 60 & 30.0 & 12 & 6.0 & 9 & 4.5 & $\mathrm{X}^{2 \mathrm{~d}}=1.309 \quad \mathrm{P}=0.519$ \\
\hline & \multicolumn{6}{|c|}{$\mathrm{X}^{2 \mathrm{a}}=1.737 \quad \mathrm{P}=0.784$} & \\
\hline \multicolumn{8}{|c|}{ Psychosocial functioning } \\
\hline - Good & 87 & 43.5 & 128 & 64.0 & 99 & 49.5 & $\mathrm{X}^{2 \mathrm{~b}}=18.27 \quad \mathrm{P}=0.000^{*}$ \\
\hline - Fair & 72 & 36.0 & 52 & 26.0 & 81 & 40.5 & $\mathrm{X}^{2 \mathrm{c}}=8.533 \quad \mathrm{P}=0.014^{*}$ \\
\hline \multirow[t]{2}{*}{ - Poor } & 41 & 20.5 & 20 & 10.0 & 20 & 10.0 & $\mathrm{X}^{2 \mathrm{~d}}=10.03 \quad \mathrm{P}=0.007^{*}$ \\
\hline & \multicolumn{6}{|c|}{$\mathrm{X}^{2 \mathrm{a}}=25.828 \quad \mathrm{P}=0.000^{*}$} & \\
\hline \multicolumn{8}{|c|}{ Total Quality of life } \\
\hline - Good & 74 & 37.0 & 121 & 60.5 & 103 & 51.5 & $\mathrm{X}^{2 \mathrm{~b}}=25.16 \quad \mathrm{P}=0.000^{*}$ \\
\hline - Fair & 86 & 43.0 & 63 & 31.5 & 80 & 40.0 & $\mathrm{X}^{2 \mathrm{c}}=14.25 \quad \mathrm{P}=0.001 *$ \\
\hline \multirow[t]{2}{*}{ - Poor } & 40 & 20.0 & 16 & 8.0 & 17 & 8.5 & $\mathrm{X}^{2 \mathrm{~d}}=3.498 \quad \mathrm{P}=0.174$ \\
\hline & \multicolumn{6}{|c|}{$\mathrm{X}^{2 \mathrm{a}}=30.202 \quad \mathrm{P}=0.000^{*}$} & \\
\hline
\end{tabular}

Figure (1 and 2): Shows that more than one tenth $(12.7 \%)$ of the studied students had internet gaming disorder. Among them, more than two fifths had mild and moderate disorder $(42.8 \%$ and $47.7 \%$ respectively), while $9.5 \%$ of the students had sever internet gaming disorder.

Figure (3): Portrays that the vast majority $(96.7 \%)$ of the studied students had computer or laptop at their homes and $82.9 \%$ of them had internet access at home. While, more than two thirds $(68.5 \%)$ of the studied

students reported having smart phones and more than one third (34.9\%) of them had internet access at their phones.

Figure (4): Portrays that more than one tenth (15.0\%) of the studied students used computer or laptop or smart phones from two to four hours per day, while $37.6 \%$ of them use it for six hours and more.

Table (1): Presents the relation between the studied students' internet gaming disorder and their basic demographic characteristics. The table reveals that internet gaming disorder was more encountered among those aged from 12 to less than 14 years (15.0\%) compared to $8.8 \%$ of those aged 16 years and more. A statistically significant relation was detected between age and internet gaming disorder $\left(\mathrm{X}^{2}=11.074, \mathrm{P}=0.004\right)$. Moreover, internet gaming disorder was more prevalence among male students than female students (13.5\% and $11.9 \%$ respectively).
A significant relation was noted between students' academic year and internet gaming disorder $\left(\mathrm{X}^{2}=143.049, \mathrm{P}=0.000\right)$. Higher percentage of internet gaming disorder was encountered among students enrolled in first preparatory grade compared to second grade students (20.0\% and $2.1 \%$ respectively). Moreover, a significant relation was observed between students' birth order and internet gaming disorder $\left(X^{2}=9.871, P=0.019\right)$, since it was more prevalent among students ranking the fourth and more $(17.5 \%)$ in comparison to those ranked as the first child within their families (10.5\%).

The same table reveals that internet gaming disorder was more prevalent among students lived in urban areas, than those who lived in rural areas $(20.9 \%$, $10.5 \%$ respectively) with a significant relation between them $\left(X^{2}=63.776, P=0.000\right)$.

Table (2): Presents the relation between the studied students' internet gaming disorder and their families' socio-demographic characteristics. The table shows that internet gaming disorder was higher among students whose mothers were died $(55.0 \%)$, followed by those whose fathers were died or divorced $(36.0 \%)$, and those whose parents were divorced $(27.7 \%)$. A statistically significant relation was existed between students' internet gaming disorder and parents' marital status $\left(\mathrm{X}^{2}=126.128, \mathrm{P}=0.000\right)$.

It is also evident from the table that internet gaming disorder was more prevalent among students with older mothers aged 45 years old and more (13.4\%) 
and those whose fathers were sixty years old and more $(25.9 \%)$. Additionally, the table shows that the age of fathers had significant impact on students' internet gaming disorder $\left(\mathrm{X}^{2}=51.853, \mathrm{P}=0.000\right)$.

It is apparent from the table that internet gaming disorder was more encountered among students whose fathers and mothers had university education (31.7\% and $22.1 \%$ respectively) compared to those whose mothers and fathers could just read and write or illiterate (1.9\% and $7.6 \%$ respectively). Moreover, the educational level of both fathers and mothers had significant impact on the students' internet gaming disorder $\left(X^{2}=159.118, \quad P=0.000\right.$ and $X^{2}=35.713$, $\mathrm{P}=0.000$ respectively).

The table also reveals that internet gaming disorder was more prevalent among students whose fathers and mothers were working $(11.4 \%$ and $28.3 \%$ respectively). A statistically significant relation existed between mothers' occupation and students' internet gaming disorder $\left(\mathrm{X}^{2}=88.060, \mathrm{P}=0.000\right)$.

The same table illustrates that internet gaming disorder was more encountered among students whose families had enough monthly income (14.8\%) and those from high socioeconomic level (23.0\%). The table also reveals that both sufficiency of family monthly income and social level had significant impact on students' internet gaming disorder $\left(\mathrm{X}^{2}=15.011, \quad \mathrm{P}=0.000\right.$ and $\mathrm{X}^{2}=64.487, \quad \mathrm{P}=0.000$ respectively).

Additionally, internet gaming disorder was higher among those who reported no parental supervision on their activities $(37.3 \%)$ with a statistically significant relation between them $\left(\mathrm{X}^{2}=452.095, \mathrm{P}=0.000\right)$.

Table (3): Shows the relation between the studied students' internet gaming disorder and their life style habits. The table portrays that internet gaming disorder was higher among students who have irregular meals per day and those who have skipped meals $(15.0 \%$ and $20.2 \%$ respectively) with statistically significant relations between them $\left(\mathrm{X}^{2}=29.017, \quad \mathrm{P}=0.000\right.$ and $\mathrm{X}^{2}=57.721, \quad \mathrm{P}=0.000$ respectively).

It is evident from the table that internet gaming disorder was more prevalent among students who did not practice physical exercises $(15.3 \%)$ than those who did $(9.7 \%)$. A statistically significant relation was observed between internet gaming disorder among students and practicing physical exercises $\left(X^{2}=14.401, P=0.000\right)$.

Lastly, it can also be observed from the table that internet gaming disorder was more encountered among students who sleep less than 7 hours per day $(13.7 \%)$ and those who reported having sleep problems $(22.3 \%)$. The table also reveals that students' internet gaming disorder had a significant relation with their sleeping problems $\left(X^{2}=121.919\right.$, $\mathrm{P}=0.000$ ).

Table (4): Presents the relation between students' internet gaming disorder and their health status. The table reveals that internet gaming disorder was higher among students who had no chronic diseases (13.6\%). Additionally, internet gaming disorder was more prevalent among students who had current health complaints (56.0\%). Among them, vision problems, neck and shoulder pain and headache were higher among students with internet gaming disorder $(58.4 \%, 52.9 \%$ and $73.6 \%$ respectively). Statistically significant relations existed between internet gaming disorder and each of presence of current health complaints and the existing complaints $\left(X^{2}=919.426\right.$, $\mathrm{P}=0.000$ and $\mathrm{X}^{2}=14.983, \mathrm{P}=0.000$ respectively).

Table (5): Presents the relation between students' internet gaming disorder and their social relation. It is apparent from the table that internet gaming disorder was higher among students who have problems in relationships with their family members, friends and school personnel $(54.3 \%, 75.0 \%$ and $60.6 \%$ respectively). Statistically significant relationships were noticed between students' internet gaming disorder and presence of problems between them and family members, friends and school personnel $\left(\mathrm{X}^{2}=55.577, \quad \mathrm{P}=0.000, \quad \mathrm{X}^{2}=758.103, \quad \mathrm{P}=0.000\right.$ and $\mathrm{X}^{2}=152.018, \mathrm{P}=0.000$ respectively).

Table (6): Illustrates the relation between students' internet gaming disorder and their academic performance. It can be observed that internet gaming disorder was higher among students who reported previous school failure (51.0\%) and among those with passable academic performance in the previous academic year $(33.7 \%)$. Statistically significant relations existed between students' internet gaming disorder and each of school failure and academic performance $\left(\mathrm{X}^{2}=537.253, \mathrm{P}=0.000\right.$ and $\mathrm{X}^{2}=244.514$, $\mathrm{P}=0.000$ respectively).

The table illustrates that internet gaming disorder was more encountered among students who were not attend to school regularly (52.8\%) with a statistically significant relation between students' internet gaming disorder and their regular attendance to school $\left(\mathrm{X}^{2}=817.993, \mathrm{P}=0.000\right)$.

It could be noticed that internet gaming disorder was more prevalent among students who were not participating in both school activities and recreational activities $(13.2 \%$ and $14.0 \%$ respectively). A statistically significant relation was detected between students' internet gaming disorder and participation in recreational activities $\left(\mathrm{X}^{2}=8.008, \mathrm{P}=0.005\right)$.

Table (7): Portrays the relation between students' internet gaming disorder and their quality of life. It is apparent from the table that among students with poor physical functioning, $34.3 \%$ of them had internet 
gaming disorder compared to $6.9 \%$ of good physical functioning, with a statistically significant relation between them $\left(\mathrm{X}^{2}=132.269, \mathrm{P}=0.000\right)$. Furthermore, among students with poor psychosocial functioning, $22.3 \%$ of them had internet gaming disorder compared to $10.3 \%$ of good psychosocial functioning, with a statistically significant relation between them $\left(\mathrm{X}^{2}=41.582, \mathrm{P}=0.000\right)$. The table also portrays that among students with poor total quality of life, $24.4 \%$ of them had internet gaming disorder compared to $9.1 \%$ of good quality of life, with a statistically significant relation between them $\left(X^{2}=53.988\right.$, $\mathrm{P}=0.000)$.

Table (8): Shows the distribution of the studied students' levels of internet gaming disorder before and after the implementation of the CBT-IA program. It was noticed that $40.0 \%$ of the students had mild internet gaming disorder, and more than half $(53.5 \%)$ had moderate level, and the rest $(6.5 \%)$ of them had sever internet gaming disorder. While, less than one third $(32.5 \%)$ of the students had no disorder immediately after the program, and those with mild and moderate level were $56 \%$ and $11.5 \%$ respectively. On the other hand, those with no disorder was $48.5 \%$ in the follow up phase, while $44.5 \%$ of the students had mild level and the rest $(7.5 \%)$ had moderate level. The same table shows that the difference between pre, post 1 and post 2 assessment was statistically significant where $\left(X^{2}=137.61, P=0.000, X^{2}=197.76, P=0.000\right.$ and $\mathrm{X}^{2}=10.885, \mathrm{P}=0.004$ respectively).

Table (9): Shows the distribution of the studied students' levels of quality of life before and after the implementation of the CBT-IA program. It was noticed that $37.0 \%$ of the students had good total quality of life, and $43.0 \%$ had fair quality of life, and the rest $(20.0 \%)$ of them had poor quality of life. While, less than two thirds $(60.5 \%)$ of the students had good total quality of life immediately after the program, and those with fair and poor quality of life were $31.5 \%$ and $8.0 \%$ respectively. On the other hand, those with good quality of life was $51.5 \%$ in the follow up phase, while $40.0 \%$ of the students had fair quality of life and the rest $(8.5 \%)$ had poor quality of life. In addition, the table shows that the difference between pre, post 1 and post 2 assessment was statistically significant where $\left(\mathrm{X}^{2}=25.16, \mathrm{P}=0.000\right.$ and $\mathrm{X}^{2}=14.25, \mathrm{P}=0.001$ respectively).

\section{Discussion}

The internet has become an important tool for social interaction, information, and entertainment. The hightech social transition towards computerization has changed the nature of recreational activities among adolescents. Nowadays, most adolescents are entertained through computer gaming, a popular and highly amusing leisure activity (Kuss, \& Griffiths, 2011). At the same time, problematic gaming, the persistent and excessive engagement in computer or video games that cannot be controlled, has been identified as a potential risk among them (Muller, et al., 2012).

Early adolescence is a distinct period of human growth and development situated between childhood and adolescence. During this remarkable stage of the life cycle, adolescents experience rapid and significant developmental change. They tend to be curious and display wide-ranging interests. Typically, they are eager to learn and explore the varied facets of their environment. They also favor active over passive experiences and prefer interactions with peers during activities (Kiraly, et al., 2014).

To make sense of the world around them, young adolescents, build upon their individual experiences and prior knowledge. So, through ease of access to the internet world, they can achieve their targets, to have fun, to learn, and to contact peers. However, the presence of relatively immature cognitive control makes this period a time of vulnerability and adjustment and may lead to a higher incidence of risky behaviors including internet gaming addiction. Overuse of internet and computer games may be a form of rebellion or sensation-seeking, providing pleasure, alleviating boredom, satisfying curiosity, facilitating social bonding, attaining peer status or as an escape/coping mechanism for this stressful period of life (Undavalli, et al., 2020).

The current study found that more than one tenth of the studied students had internet gaming disorder and among them, it was more encountered among younger students in comparison to older students. These findings come in line with the results of (Muller, et al., 2012, \& Kheradmand, et al., 2012) who found that less than one fifth of the young adolescents suffered from computer games addiction.

Previous studies identified gender as a risk factor for internet gaming disorder as boys are more addicted and spend more time playing on computers than girls (Festl, et al., 2012). The current study found that internet gaming disorder was more prevalent among male students. One explanation for this may be that males have a stronger motivation to play and pick up more positive thoughts from playing computer games, and thus, computers are regarded as a "male toy".

Moreover, the current study found that internet gaming disorder was less encountered among the first-born child. Similar findings were reported by (Kuss, \& Griffiths, 2011, \& Gnetile, et al., 2011). This finding could be attributed to that the first-born child is the center of attention of his family before a new sibling is incorporated into the family. The first born is "an only child" before another sibling is born 
parents heavily invest in their first-born child. While, the last born has the least amount of care, time and attention of their parents, so they may engage deeply into the computer games world as a defense mechanism to attaining a status or as an escape/coping mechanism. Furthermore, the current study found that internet gaming disorder was higher among students who live in urban areas. This could be attributed to that urban areas may have better chance of high-speed internet access than rural areas. In addition, the living environment has a great impact over the lifestyles of children, urban children because of geographical constraints tend to stay more at home and involved in multimedia, on the other hand the chance of outdoor gaming is more among rural children. In the same line, Undavalli, et al., (2020) who found that the majority of the study participants with gaming disorder were from urban area.

Family and home environment are detrimental factors in adolescents' life. Age, level of education and occupation of parents are considerable familial factors in a wide variety of risk-taking behaviors among adolescents. The present study found that internet gaming disorder was more prevalent among students whose parents were older, highly educated and working. Those older parents didn't pay much attention to behaviors of their children/off springs and have less control, care and supervision than the younger parents. While, working parents are busy at work and have less time to spend with their adolescents to monitor them. Moreover, highly educated parents have better chances using modern technology and gadgets, which increased the adolescents' access to internet. In the same line, the results of Shek, et al., (2015) who found an association between the parents' age, occupation and level of education and the development of internet gaming disorder among their children.

Additionally, the current study found that internet gaming disorder was higher among those students whose parents were died or divorced. This finding could be attributed to that a broken home is associated with poor family functioning, lower parental control and care and higher stress level. So, the adolescents may forget their stress during internet access hours as a form of escape. Similar findings were reported by (Bonnaire, \& Phan, 2017) who found that unstable home environment was significantly associated with internet gaming disorder among adolescents.

Problematic gaming may lead to several negative psychosocial consequences and mental health problems affecting work, education, family, friends, social life, psychosocial well-being, social competence, leisure activities, self-esteem, and loneliness (Shek, et al., 2015). The same picture was portrayed in the current study findings as internet gaming disorder was higher among those students with poor psychosocial quality of life and those who reported problems in relations with family, friends and school personnel. Similar results were reported by Lemmens, et al., (2011).

This could be attributed to the fact that the online gaming may temporarily offer social, recreational and personal gratification but excessive engagement can backfire to addictive behaviors entailing several pathological behavioral outcomes. Spending excessive time on online gaming hinders the accumulation of stable real-life friendships and the development of social skills. Social interaction is displaced to superficial and short-term online interactions, which produces deterioration of present real-life interactions and in turn increases social isolation and magnifies feelings of loneliness.

Another explanation is that the virtual social environment created through digital games enables the gamer to interact with people having similar gaming interests. This rather reinforces gaming instead of criticizing it. Virtual relationships thus become increasingly more important for the gamer, and virtual rank and status increasingly satisfy otherwise deficient social needs and reinforce online presence. Moreover, the guild or clan may exert social pressure to attach gamers to the game and keep them active. Gamers will receive social rewards for gaming skills in the real peer group as well. Pathological gamers tend to overestimate gaming rewards, activities, virtual identities, or hard-earned 'valuable' items (weapons, life) which in turn increase the time spend online.

Moreover, late night use of the internet can cause sleep deprivation and fatigue, which can adversely affect academic performance and school attendance. In the long term, it leads to sedentary life style and can cause serious health problems such as repetitive strain injury and back ache (Lemmens, et al., 2011). Similarly, the current study findings revealed that internet gaming disorder was more common among those who skipped meals, had irregular meal time, not practicing physical exercises, those with less sleeping hours and had sleeping problems and those with poor physical quality of life.

In addition, the current study reveals that vision problems, neck, shoulder pain and headache were more encountered among students with internet gaming disorder. In the same line, results of (Kawabe, et al., 2019 \& Toker, \& Baturay, 2016) found that adolescents with problematic internet use were more likely to suffer from somatic complaints could derive from spending prolonged hours on the computer screen and as a result having eye problems, 
dizziness, headaches and sleep disturbances. Moreover, somatic complaints may have given rise to sleep deprivation, due to excessive engagement in online gaming during the late evening hours, is related to hallucinations.

Cognitive-behavioral therapy (CBT) has long been advocated as a treatment and has been extended to reduce internet addiction in the past decades. According to CBT, an event cannot directly lead to emotional and behavioral disorders. What really important is one's understanding and evaluation of the event. It uses cognitive reconstruction to correct people's negative thoughts, helping individuals get a new meaning and improve their thoughts, feelings, and behaviors (Dong, \& Potenza, 2014).

Internet gaming addiction is a complex and gradual habit which is acquired under the combined action of defective cognition and behavior. The correction of this behavior requires the change of cognitive style, correction of behavior habits, and the maintenance of state after rehabilitation. Its therapeutic objectives generally include cognition, emotion, and behavior, through cognitive reconstruction, skill-building, and lifestyle reorganization (Dong, \& Potenza, 2014).

Cognitive behavioral therapy aims to help those with internet gaming disorders to become aware of thought distortion which in turn causing psychological distress, and of behavioral patterns which are reinforcing it, and to correct them. Throughout this process, the adolescents acquire coping strategies as well as improved skills of appropriate decision making, critical thinking, assertiveness and communication skills (Young, \& Brand, 2017 \& Zajac, et al., 2017).

The efficacy of the cognitive behavioral therapy was portrayed in the current study findings, where the percentage of normal students with no disorder has been raised to $32.5 \%$ and $48.5 \%$ in the immediate evaluation and in the follow up phase after three months respectively. These results came in accordance with several studies which found that CBT was one of the most effective treatment strategies for treatment of internet addiction and internet gaming disorder. In the same line, the results of (Wolfling, et al., 2019, Torres Rodriguez, et al., 2018 \& Han, et al., 2020) who proved the ability of cognitive behavioral therapy to target and modify maladaptive cognitions that underlie gaming behaviors that generate harm and/or distress among the studied groups. Another benefit of cognitive behavioral therapy is that it may be more equipped to address comorbid conditions in the context of internet gaming disorder. Adolescents with internet gaming disorder are more likely to have some critical impairment in their quality of life, such as higher prevalence of mood and anxiety disorders, poor sleep quality, pain, muscle fatigue and poor academic performance (Machimbarrena, et al., 2019). In The same line, findings of the current study found that the percentage of students with good quality of life increased to $60.5 \%$ immediately after the program and to $51.5 \%$ in the follow up phase.

\section{Conclusion}

Based upon the findings of the current study, it could be concluded that, internet gaming disorder is a prevalent problem among preparatory school students. The cognitive behavioral therapy positively affects each of student's internet gaming disorder and their quality of life.

\section{Recommendations}

In line with the findings of the study, the following recommendations are made:

- Perform a regular screening for internet gaming disorder among children and adolescents.

- Encourage face-to-face socialization and healthy social relationships.

- Encourage a healthy life style for adolescents such as adequate amount of sleep, exercise, and a balanced diet.

- Encourage parents to monitor their children's hours and purpose of internet usage help in controlled internet use and gaming disorders.

- Develop a guideline for interventions to address internet gaming disorder among adolescents at the primary health-care level.

- Early interventions may include counseling regarding monitoring of internet use either by self or involving family members.

\section{References}

- Al Gammal, M., Elsheikh, M., Abozahra, A.. (2019). Internet Addiction and Internet Gaming Disorder and Associated Insomnia among a Sample of Al-Azhar University Students, Clinical Study. The Egyptian Journal of Hospital Medicine, 77(5), 5718-5726. doi: 10.12816/ejhm.2019.63227

- Bonnaire, C., \& Phan, O., (2017): Relationships between parental attitudes, family functioning and internet gaming disorder in adolescents attending school. Psychiatry Research, 255, 104110. https://doi.org/10.1016/j.psychres.2017.05.030

- Chen, X., Origasa, H., Ichida, F., Kamibeppu, K., \& Varni, J., (2007): Reliability and validity of the Pediatric Quality of Life Inventory ${ }^{\mathrm{TM}}$ (PedsQL ${ }^{\mathrm{TM}}$ ) Short Form 15 Generic Core Scales in Japan. Quality of Life Research, 16(7), 12391249. https://doi.org/10.1007/s11136-007-9230-4

- Dong, G., \& Potenza, M., (2014): A cognitivebehavioral model of internet gaming disorder: Theoretical underpinnings and clinical 
implications. Journal of Psychiatric Research, 58, 7 11. https://doi.org/10.1016/j.jpsychires.2014.07.005

- Festl, R., Scharkow, M., \& Quandt, T., (2012): Problematic computer game use among adolescents, younger and older adults. Addiction, 108(3), 592 599. https://doi.org/10.1111/add.12016

- Gentile, D., Choo, H., Liau, A., Sim, T., Li, D., Fung, D., (2011): Pathological video game use among youths: A two-year longitudinal study. PEDIATRICS, 127(2), e319e329. https://doi.org/10.1542/peds.2010-1353

- Han, J., Seo, Y., Hwang, H., Kim, S., \& Han, D., (2020). Efficacy of cognitive behavioral therapy for internet gaming disorder. Clinical Psychology \& Psychotherapy, 27(2),

$203-$ 213. https://doi.org/10.1002/cpp.2419

- Hawi, N., \& Samaha, M., (2017): Validation of the Arabic version of the internet gaming disorder-20 test. Cyberpsychology, Behavior, and Social Networking, 20(4), 268-

272. https://doi.org/10.1089/cyber.2016.0493

- Kawabe, K., Horiuchi, F., Oka, Y., \& Ueno, S. (2019): Association between sleep habits and problems and internet addiction in adolescents. Psychiatry Investigation, 16(8), 581587. https://doi.org/10.30773/pi.2019.03.21.2

- Kheradmand, A., Zamani, B., Hedayati, N., Cheshomi, M., \& Abedi, A., (2012): P-45 Comparing the social skills of students addicted to computer games with normal students. European Psychiatry, 27, 1. https://doi.org/10.1016/s09249338(12)74212-8

- Király, O., Griffiths, M. D., Urbán, R., Farkas, J., Kökönyei, G., Elekes, Z., Tamás, D., \& Demetrovics, Z., (2014): Problematic internet use and problematic online gaming are not the same: Findings from a large nationally representative adolescent sample. Cyberpsychology, Behavior, and Social Networking, 17(12), 749754. https://doi.org/10.1089/cyber.2014.0475

- Kuss, D., \& Griffiths, M., (2011): Internet gaming addiction: A systematic review of empirical research. International Journal of Mental Health and Addiction, 10(2), $278-$ 296. https://doi.org/10.1007/s11469-011-9318-5

- Kuss, D., \& Griffiths, M., (2015): Internet addiction: Risk. Internet Addiction in Psychotherapy, $15-$ 53. https://doi.org/10.1057/9781137465078_3

- Lee, G., \& Morgan, H., (2018): Understanding children's attraction toward digital games and preventing their gaming addiction. US-China Education Review A, 8(1), 11-17. doi: $10.17265 / 2161-623 X / 2018.01 .002$
- Lee, J., \& Kang, G., (2015): A meta-analysis on the effect of youth internet addiction group counseling program. Korea Journal of Counseling, 16(3), $101-$ 120. https://doi.org/10.15703/kjc.16.3.201506.101

- Lemmens, J., Valkenburg, P., \& Peter, J., (2011): Psychosocial causes and consequences of pathological gaming. Computers in Human Behavior, 27(1), 144152. https://doi.org/10.1016/j.chb.2010.07.015

- Machimbarrena, J., González-Cabrera, J., Ortega-Barón, J., Beranuy-Fargues, M., Álvarez-Bardón, A., \& Tejero, B., (2019): Profiles of problematic internet use and its impact on adolescents' health-related quality of life. International Journal of Environmental Research and Public Health, 16(20), 3877. https://doi.org/10.3390/ijerph16203877

- Muller, K., Freisleder, F., Beutel, M., Wolfling, K., (2012): Addictive Internet use as a comorbid disorder among clients of an adolescent psychiatry prevalence and psychopathological symptoms. Z Kinder Jugendpsychiatr Psychother, 0: 331-7. https:// doi.org/10.1024/1422-4917/a000190

- Müller, K., Janikian, M., J J J Wölfling, K., $\quad$ Beutel, M., $\quad$ Tzavara, C., Richardson, C., \& Tsitsika, A., (2014): Regular gaming behavior and internet gaming disorder in European adolescents: Results from a cross-national representative survey of prevalence, predictors, and psychopathological correlates. European Child \& Adolescent Psychiatry, 24(5), 565574. https://doi.org/10.1007/s00787-014-0611-2

- Pontes, H., Király, O., $\quad$ Demetrovics, Z., \& Griffiths, M., (2014): The conceptualisation and measurement of DSM-5 internet gaming disorder: The development of the IGD-20 test. PLoS ONE, 9(10),

e110137. https://doi.org/10.1371/journal.pone.0110 137

- Potenza, M., (2015): Internet addiction: diagnostic and classification considerations in the time of DSM-5 and ICD-11. J Behav Addict. 4:31. https://doi.org /10.3389/fpsyt.2019.00405

- Reda, M., Rabie, M., Mohsen, N., \& Hassan, A., (2012): Problematic internet users and psychiatric morbidity in a sample of Egyptian adolescents. Psychology, 03(08), 626631. https://doi.org/10.4236/psych.2012.38096

- Shek, D., Xie, Q., \& Lin, L., (2015): The impact of family intactness on family functioning, parental control, and parent internet addiction and internet child relational qualities in a Chinese context. Frontiers in Pediatrics, 2. https://doi.org/10.3389/fped.2014.001 49 
- Stevens, M., King, D., $\quad$ Dorstyn, D., $\quad$ \& Delfabbro, P., (2018): Cognitive-behavioral therapy for internet gaming disorder: A systematic review and meta-analysis. Clinical Psychology \& Psychotherapy, 26(2),

191-

203. https://doi.org/10.1002/cpp.2341

- Toker, S., \& Baturay, M., (2016): Antecedents and consequences of game addiction. Computers in Human Behavior, 55, 668679. https://doi.org/10.1016/j.chb.2015.10.002

- Torres-Rodríguez, A.,

Griffiths, M., Carbonell, X., \& Oberst, U., (2018): Treatment efficacy of a specialized psychotherapy program for internet gaming disorder. Journal of Behavioral Addictions, 7(4), 939952. https://doi.org/10.1556/2006.7.2018.111

- Undavalli, V., Rani, G., \& Kumar, J., (2020): Prevalence of internet gaming disorder in India: A technological hazard among adolescents. International Journal of Community Medicine and Public Health, 7(2), 688. https://doi.org/10.18203/23946040.ijcmph20200450

- Weinstein, A., Curtiss Feder, L., Rosenberg, K., \& Dannon, P., (2014): Internet addiction disorder. Behavioral Addictions, 99117. https://doi.org/10.1016/b978-0-12-4077249.00005-7

- Wichstrom, L., Stenseng, F., Belsky, J., Von Soest, T., \& Hygen, B., (2018): Symptoms of internet gaming disorder in youth: Predictors and comorbidity. Journal of Abnormal Child Psychology, 47(1), 71 83. https://doi.org/10.1007/s10802-018-0422-x

- Wölfling, K., $\quad$ Beutel, M., $\quad$ Dreier, M., $\quad \&$ Müller, K., (2014): Treatment outcomes in patients with internet addiction: A clinical pilot study on the effects of a cognitive-behavioral therapy program. BioMed Research International, 2014, 18. https://doi.org/10.1155/2014/425924

- Wölfling, K., Müller, K. W., Dreier, M., Ruckes, C., Deuster, O., Batra, A., Mann, K., Musalek, M., Schuster, A., $\quad$ Lemenager, T., Hanke, S., \& Beutel, M., (2019): Efficacy of shortterm treatment of internet and computer game addiction. JAMA Psychiatry, 76(10), 1018. https://doi.org/10.1001/jamapsychiatry.2019. 1676

- Young, K., (2011): CBT-IA: The first treatment model for internet addiction. Journal of Cognitive Psychotherapy, 25(4), 312. https://doi.org/10.1891/0889-8391.25.4.304

- Young, K., \& Brand, M., (2017): Merging theoretical models and therapy approaches in the context of internet gaming disorder: A personal perspective. Frontiers
Psychology, 8. https://doi.org/10.3389/fpsyg.2017.0 1853

- Zajac, K., Ginley, M., Chang, R., \& Petry, N., (2017): Treatments for internet gaming disorder and internet addiction: A systematic review. Psychology of Addictive Behaviors, 31(8), 979994. https://doi.org/10.1037/adb0000315 